\title{
Diabetic cardiomyopathy: a hyperglycaemia- and insulin-resistance-induced heart disease
}

\author{
Guanghong Jia ${ }^{1,2}$ • Adam Whaley-Connell ${ }^{1,2,3}$ • James R. Sowers ${ }^{1,2,4,5}$
}

Received: 17 April 2017 / Accepted: 13 June 2017 / Published online: 3 August 2017

(C) Springer-Verlag GmbH Germany 2017

\begin{abstract}
Diabetic cardiomyopathy is characterised in its early stages by diastolic relaxation abnormalities and later by clinical heart failure in the absence of dyslipidaemia, hypertension and coronary artery disease. Insulin resistance, hyperinsulinaemia and hyperglycaemia are each independent risk factors for the development of diabetic cardiomyopathy. The pathophysiological factors in diabetes that drive the development of cardiomyopathy include systemic metabolic disorders, inappropriate activation of the renin-angiotensin-aldosterone system, subcellular component abnormalities, oxidative stress, inflammation and dysfunctional immune modulation. These abnormalities collectively promote cardiac tissue interstitial fibrosis, cardiac stiffness/diastolic dysfunction and, later, systolic dysfunction, precipitating the syndrome of clinical heart failure. Recent evidence has revealed that dysregulation of coronary endothelial
\end{abstract}

Electronic supplementary material The online version of this article (doi:10.1007/s00125-017-4390-4) contains a slideset of the figures to download, which is available to authorised users.

Guanghong Jia

Jiag@health.missouri.edu

$\triangle$ James R. Sowers

Sowersj@health.missouri.edu

1 Diabetes and Cardiovascular Research Center, University of Missouri School of Medicine, D109 Diabetes Center HSC, One Hospital Drive, Columbia, MO 65212, USA

2 Research Service, Harry S Truman Memorial Veterans Hospital, Research Service, Columbia, MO, USA

3 Division of Nephrology and Hypertension, Department of Medicine, University of Missouri School of Medicine, Columbia, MO, USA

4 Department of Medical Pharmacology and Physiology, University of Missouri School of Medicine, Columbia, MO, USA

5 Dalton Cardiovascular Research Center, University of Missouri, Columbia, MO, USA cells and exosomes also contributes to the pathology behind diabetic cardiomyopathy. Herein, we review the relationships among insulin resistance/hyperinsulinaemia, hyperglycaemia and the development of cardiac dysfunction. We summarise the current understanding of the pathophysiological mechanisms in diabetic cardiomyopathy and explore potential preventative and therapeutic strategies.

Keywords Cardiac dysfunction · Heart failure . Hyperglycaemia $\cdot$ Insulin resistance $\cdot$ Review

$\begin{array}{ll}\text { Abbreviations } & \\ \text { AMPK } & \begin{array}{l}\text { Adenosine monophosphate-activated } \\ \text { protein kinase } \\ \text { Coronary heart disease }\end{array} \\ \text { DPP-4 } & \text { Dipeptidyl peptidase-4 } \\ \text { EDHF } & \text { Endothelium-derived hyperpolarising } \\ & \text { factor } \\ \text { ER } & \text { Endoplasmic reticulum } \\ \text { GLP-1 } & \text { Glucagon-like peptide 1 } \\ \text { mTOR } & \text { Mammalian target of rapamycin } \\ O-G l c N A c y l a t i o n & O-N \text {-Acetylglucosamine acylation } \\ \text { PGC-1 } \alpha & \text { PPAR- } \gamma \text { coactivator-1 } \alpha \\ \text { PKC } & \text { Protein kinase C } \\ \text { PKG } & \text { Protein kinase G } \\ \text { PPAR- } \alpha & \text { Peroxisome proliferator-activated } \\ \text { PPAR- } \gamma & \text { receptor- } \alpha \\ \text { PI3K } & \text { Peroxisome proliferator-activated } \\ \text { RAGE } & \text { receptor- } \gamma \\ \text { ROS } & \text { Phosphoribosyls 3-kinase } \\ \text { RAAS } & \text { AGE receptor } \\ \text { S6K1 } & \text { Reactive oxygen species } \\ & \text { Renin-angiotensin-aldosterone system } \\ & \text { S6 kinase 1 }\end{array}$




\section{Introduction}

Diabetic cardiomyopathy is a specific form of heart disease, promoted by resistance to the metabolic actions of insulin in heart tissue (e.g. insulin resistance), compensatory hyperinsulinaemia and the progression of hyperglycaemia, which occurs independent of other cardiac risk factors such as coronary artery disease (CAD) and hypertension. Diabetic cardiomyopathy as a distinct entity was first described in 1972 in four individuals with diabetes who manifested heart failure symptoms [1]. This was confirmed in a secondary analysis of the Framingham Heart Study in 1974, which found that the risk of heart failure was increased 2.4-fold in men and fivefold in women with diabetes compared with individuals without diabetes after adjustment for other risk factors including age, hypertension, obesity, dyslipidaemia and CAD [2]. By ruling out CAD using angiography, a study of 17 individuals with type 2 diabetes in 1977 provided definitive evidence of diabetic cardiomyopathy characterised by increased cardiac left ventricular end-diastolic pressure, decreased left ventricular compliance and low left ventricular ejection fraction with diffuse hypokinesis. These initial observations suggested that diabetes had a distinct and direct impact on interstitial fibrosis and associated reduced left ventricular compliance and diastolic dysfunction [3]. In this context, the clinical course of cardiac dysfunction in diabetes progresses from subclinical cardiac abnormalities, such as left ventricular fibrosis and diastolic dysfunction, to severe diastolic heart failure with normal ejection fraction and eventually to systolic dysfunction accompanied by heart failure with reduced ejection fraction $[4,5]$. This review summarises recent work in diabetic cardiomyopathy and examines the molecular mechanisms involved in this hyperglycaemia- and insulin-resistance-induced cardiac dysfunction.

\section{The prevalence of diabetic cardiomyopathy associated with type 1 and type 2 diabetes}

Cardiac diastolic dysfunction is more common than systolic dysfunction in people with type 1 diabetes [6]. In an observational study of 20,985 individuals with type 1 diabetes (mean age 38.6 years), each $1 \%$ increase in $\mathrm{HbA}_{1 \mathrm{c}}$ (compared with earlier levels) was associated with a $30 \%$ increase in risk of heart failure, independent of other risk factors including hypertension, smoking and obesity [7]. Further data derived from a population-based observational study involving children and adolescents (8-18 years old) with type 1 diabetes, echocardiographic Doppler imaging confirmed that there was a lower ratio of early $\left(\mathrm{E}^{\prime}\right)$ to late $\left(\mathrm{A}^{\prime}\right)$ peak diastolic velocities in all measurements from the mitral and lateral tricuspid annulus ring. These data suggest that cardiac diastolic dysfunction is present in early stages of type 1 diabetes in children and adolescents despite treatment with intensified insulin regimens [8]. Another cross-sectional study involving middleaged people with type 1 diabetes suggested that there is both left and right ventricular systolic impairment as assessed by isovolumetric contraction in Doppler imaging [9]. These collective data demonstrate that individuals with type 1 diabetes have a high risk of developing diastolic dysfunction that evolves into clinical heart failure independent of their $\mathrm{HbA}_{1 \mathrm{c}}$, blood pressure or lipid control.

The risk for development of cardiac stiffness/diastolic dysfunction is also high in those with type 2 diabetes [10]. For example, observations from the Framingham Heart Study indicated that people with type 2 diabetes had a two- to eightfold increased risk for development of heart failure and that $19 \%$ developed symptoms of heart failure [2]. A retrospective cohort study of 8231 individuals with type 2 diabetes indicated that heart failure developed in 30.9 out of 1000 people, compared with an incidence of 12.4 per 1000 in individuals without diabetes, indicating a 2.5 -fold increase in heart failure risk in those with type 2 diabetes [11]. Furthermore, an observational study involving 25,958 men and 22,900 women with type 2 diabetes indicated that a $1 \%$ increase in of $\mathrm{HbA}_{1 \mathrm{c}}$ was associated with an $8 \%$ increase in the risk of heart failure, independent of blood pressure, obesity, age and the presence of CAD, suggesting that type 2 diabetes is an independent risk for incident heart failure [12]. Conversely, in a prospective observational study, each $1 \%$ reduction in $\mathrm{HbA}_{1 \mathrm{c}}$ level was associated with a $16 \%$ reduction in risk for heart failure, $14 \%$ reduction in risk for myocardial infarction and $21 \%$ reduction in risk for deaths related to type 2 diabetes [13]. These data support the notion that glycaemic control in those with type 2 diabetes is a critical mechanism in the prevention of cardiac dysfunction and heart failure.

\section{Changes in cardiac structure and function in diabetic cardiomyopathy}

In the early stages of diabetic cardiomyopathy, the cardiac structural and functional adaptations are promoted by metabolic disturbances including impaired insulin metabolic signalling, excess ambient insulin, impaired glucose uptake, increases in myocardial NEFA uptake and mitochondrial dysfunction [4, 14]. These collective metabolic disturbances promote cardiac remodelling, fibrotic diastolic dysfunction and, ultimately, decreased ejection fraction in those with diabetes $[14,15]$. In this regard, the pathophysiological changes, including impaired cardiomyocyte autophagy, increased cardiomyocyte death, inappropriate renin-angiotensin-aldosterone system (RAAS) activation despite salt and water excess, oxidative stress and maladaptive immune responses, result in fibrosis and substantial cardiac stiffness/diastolic dysfunction [14, 15]. Our recent investigations also show that consumption of a diet high in fat 
and refined carbohydrates induces impaired cardiac insulin metabolic signalling, inflammation, oxidative stress, maladaptive immune modulation, reduced bioavailable NO, increased connective tissue crosslinking and fibrosis [5]. These alterations result in diastolic dysfunction characterised by slow initial and peak filling rates, as demonstrated by cine magnetic resonance imaging [5]. In the later stages of diabetic cardiomyopathy, the alterations in cardiac structure are more pronounced and include cardiomyocyte necrosis, collagen accumulation, increased crosslinking of connective tissue, interstitial fibrosis, hypertrophy, sclerotic small coronary vessels and capillary microaneurysms [16, 17]. These more advanced changes are associated with coronary microcirculation dysfunction and both diastolic and systolic dysfunction. Therefore, consumption of a contemporary diet high in fat and refined carbohydiabetic cardiomyopathy.

\section{Pathophysiological mechanisms involved in the development of diabetic cardiomyopathy}

Abnormal insulin metabolic signalling Impaired insulin metabolic signalling in the heart is a key pathophysiological abnormality associated with diabetic cardiomyopathy (Fig. 1). One instigating pathway associated with this impaired signalling is the mammalian target of rapamycin (mTOR)-S6 kinase nutrient sensor and modulates insulin metabolic signalling drates can lead to cardiac insulin resistance and an early form of 1 (S6K1) pathway [4]. This pathway is a highly conserved

through increased phosphorylation of S6K1, an evolutionarily conserved serine (Ser) kinase [4]. Evidence is mounting that chronic activation of S6K1, by both excessive nutrient intake and inappropriate activation of the RAAS [18], promotes insulin resistance in heart, fat, liver and skeletal muscle tissue through increased Ser phosphorylation of the critical insulin signalling/docking molecule IRS-1. This then impairs phosphoinositol 3-kinase (PI3K) engagement and protein kinase B (Akt) stimulation [4, 5]. Under normal physiological conditions in cardiomyocytes, the PI3K/Akt signalling pathway stimulates GLUT4 recruitment to the plasma membrane, resulting in glucose uptake into the heart [4] (Fig. 2). The reduction of glucose uptake resulting from PI3K/Akt impairment decreases $\mathrm{Ca}^{2+}$ ATPase activity and moves $\mathrm{Ca}^{2+}$ back into the sarcoplasmic reticulum, thus increasing intracellular $\mathrm{Ca}^{2+}$ [4]. In addition, impairment of insulin metabolic signalling inhibits cardiac insulin-stimulated coronary endothelial NO synthase activity and NO production, which further increases intracellular $\mathrm{Ca}^{2+}$ levels and $\mathrm{Ca}^{2+}$ sensitisation in cardiomyocytes via the cGMP/PKG signalling pathway [4]. These abnormalities cause cardiac stiffness and diastolic dysfunction (Fig. 2). Our research group has observed that overnutrition/RAAS activation of mTOR-S6K1 pathway and the consequent diminution of insulin metabolic signalling is associated with cardiac fibrosis/stiffness and diastolic dysfunction [18].

Hyperglycaemia and glucotoxicity Hyperglycaemia and glucotoxicity can induce a protein glycation reaction leading

Fig. 1 Pathophysiological mechanisms of diabetic cardiomyopathy. Hyperglycaemia and insulin resistance induce increases in AGEs, cardiac lipotoxicity, activation of the RAAS, coronary endothelial dysfunction and dysregulation of exosomes, which, in turn, result in mitochondrial dysfunction, oxidative stress, ER stress and impairment of calcium homeostasis. These pathophysiological abnormalities are associated with cardiac hypertrophy, fibrosis, death, stiffness, diastolic dysfunction and heart failure

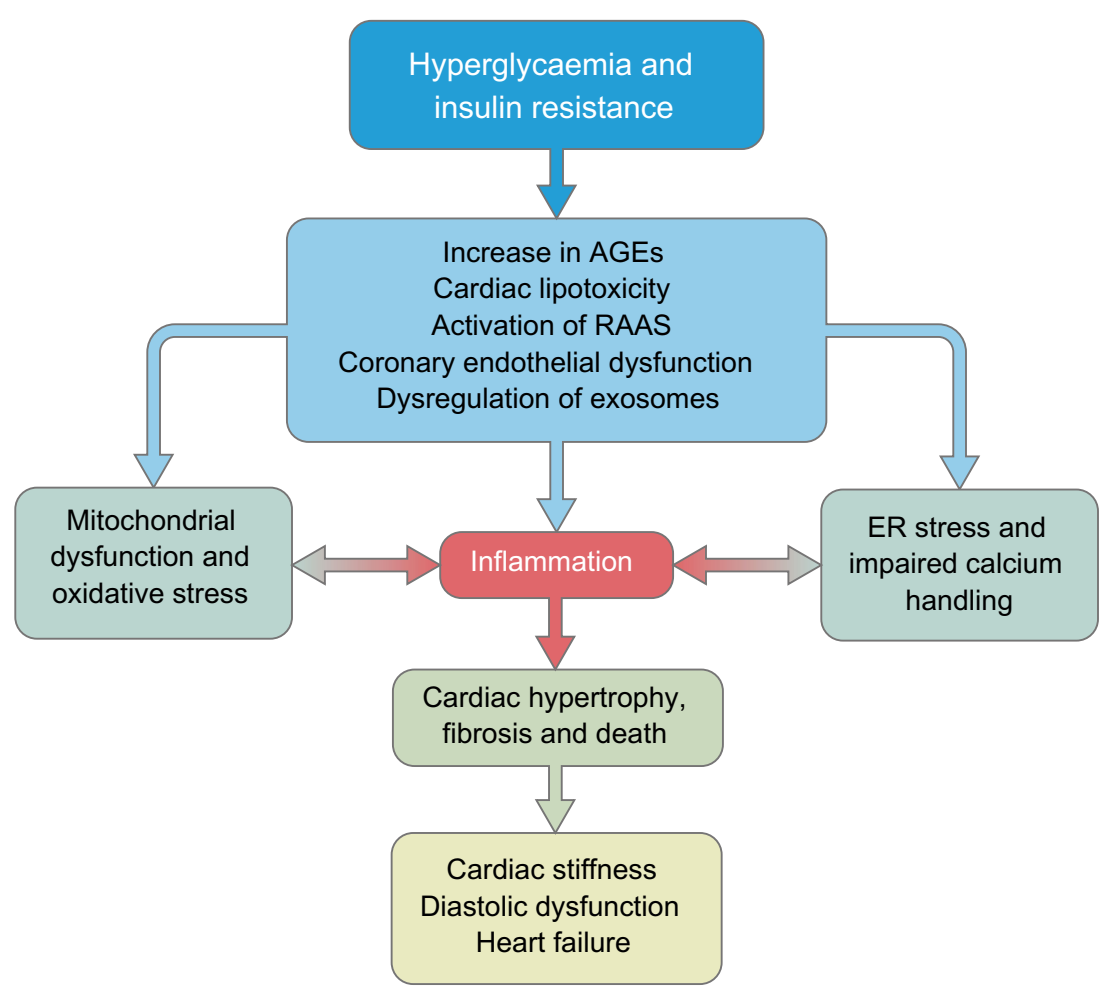




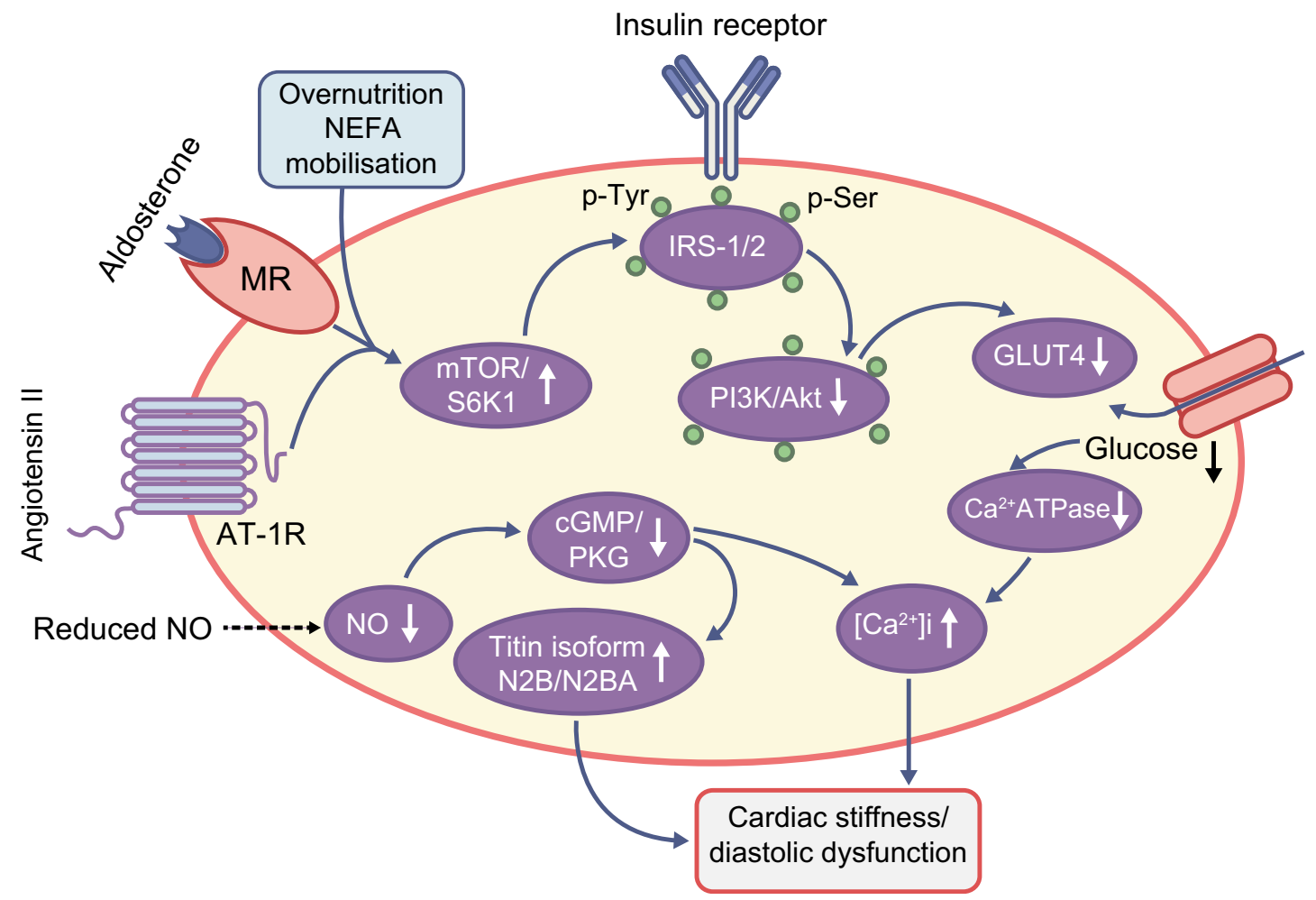

Fig. 2 Insulin metabolic signalling in diabetic cardiomyopathy. In cardiac muscle cells, overnutrition, NEFA mobilisation, aldosterone and angiotensin II impair cardiac insulin metabolic signalling of PI3K/Akt through activation of mTOR/S6K1 and phosphorylation of IRS-1/2. Reduction in NO increases the ratio of titin isoform N2B/N2BA, intracellular $\mathrm{Ca}^{2+}$, and $\mathrm{Ca}^{2+}$ sensitisation in cardiomyocytes by inhibition of cGMP/PKG. Impairment of insulin metabolic signalling also inhibits

to increases in AGEs, which are produced from non-enzymatic glycosylation of lipids, lipoproteins and amino acids. AGE deposition contributes to increased connective tissue crosslinking, fibrosis, cardiac stiffness and impaired diastolic relaxation [4] (Fig. 1). For example, elevated serum levels of AGEs are associated with a prolongation of left ventricular relaxation time in individuals with early diabetic heart failure [19]. Indeed, AGEs induce changes in mechanical properties of the extracellular matrix by increasing resistance to connective tissue enzymatic proteolysis and enhancing the crosslinking of collagens and laminins; these changes lead to increased fibrosis, reduced cardiac compliance and left ventricular diastolic dysfunction [20]. AGEs may bind to the cell surface receptor for AGE (RAGE) to prompt maladaptive inflammatory gene expression, thus increasing matrix proteins via processes mediated through mitogen-activated protein kinase (MAPK) and Janus kinase (JAK) pathways in vascular and cardiac tissues [4]. Meanwhile, AGEs are involved in increasing the production of reactive oxygen species (ROS), which promote inflammation and fibrosis [4]. To this point, in a mouse model of type 1 diabetes, administration of a RAGE antagonist prevented AGEs/RAGE signalling-mediated increases in myocardial collagen, fibrosis, stiffness and diastolic dysfunction [21].
GLUT4 recruitment to the plasma membrane and subsequently glucose uptake into the heart, which decreases $\mathrm{Ca}^{2+}$ ATPase activity and thus increases intracellular $\mathrm{Ca}^{2+}$. These abnormalities cause cardiac stiffness and diastolic dysfunction. AT-1R, angiotensin II receptor 1; MR, mineralocorticoid receptor; $\left[\mathrm{Ca}^{2+}\right] \mathrm{i}$, intracellular calcium; ATPase, adenosine triphosphatase

Therefore, hyperglycaemia, glucotoxicity and associated activation of AGEs/RAGE signalling are important contributors in the development of the myocardial fibrosis that is an integral part of diabetic cardiomyopathy.

The maladaptive hyperglycaemia-associated hexosamine biosynthesis pathway and $\mathrm{O}-\mathrm{N}$-acetylglucosamine acylation (O-GlcNAcylation) pathway have also been found to be involved in heart disease in individuals with diabetes [22]. For example, chronically increased $O$-GlcNAcylation induces mitochondrial dysfunction and impairment of left ventricular function [22]. Overexpression of $O$-linked $N$-acetylgluco saminyl hydrolase removes $O$-linked $N$-acetylglucosamine to restore normal cardiomyocyte $\mathrm{Ca}^{2+}$ handling and cardiac function [23], suggesting that targeting hexosamine biosynthesis and $O$-GlcNAcylation might be a potential therapeutic strategy in the prevention of diabetic cardiomyopathy.

Cardiac lipotoxity Abnormal lipid metabolism often accentuates the process of diabetic cardiomyopathy (Fig. 1). Increased circulating triacylglycerol levels lead to increased fatty acid delivery to cardiomyocytes and, thus, enhanced fatty acid $\beta$-oxidation and impaired insulin metabolic signalling in diabetic hearts. Further, expression of fatty acid transporters 
such as CD36 is increased in diabetic hearts [24]. CD36, a transporter protein involved in fatty acid uptake, is present on both sarcolemmal and endosomal membranes [24]. The rate of cellular fatty acid uptake is modulated through the subcellular vesicular recycling of CD36 from endosomes to the plasma membrane, and this is increased in diabetic hearts [4, 24]. Additionally, in diabetes, the heart often displays increased expression of peroxisome proliferator-activated receptor- $\alpha$ $($ PPAR- $\alpha)$, associated with increased fatty acid uptake, triacylglycerol accumulation and reduced glucose utilisation [25]. Some lipid metabolites, such as diacylglycerols and ceramides, impair insulin metabolic signalling further promoting diabetic cardiomyopathy. Increased levels of diacylglycerols in cardiomyocytes impair glucose metabolism through activation of protein kinase $\mathrm{C}$ (PKC) isoforms thereby reducing insulin metabolic signalling and NO production [26]. Ceramides are a family of bioactive waxy lipid molecules composed of sphingosine and a fatty acid [26]. Ceramide directly activates atypical PKCs to phosphorylate and inhibit the insulin metabolic Akt signalling; thus, increased ceramides in diabetic hearts attenuates GLUT4 translocation and insulinstimulated glucose uptake [26]. Therefore, excess accumulation of lipids and lipid metabolites contributes to cardiac insulin resistance, reduced bioavailable NO, inflammation, fibrosis and diastolic dysfunction. Recently, one study suggested that individuals with diabetes possess an inherent metabolic advantage whereby the failing myocardium is able to effectively use ketone bodies as a source of fuel [27]. This notion has been confirmed in a study in which empagliflozin, a sodium-glucose cotransporter 2 (SGLT2) antagonist, elevated the ketone body levels in individuals with diabetes and heart failure [27].

Mitochondrial dysfunction and oxidative stress The increased mitochondrial fatty acid uptake and $\beta$-oxidation in diabetic hearts may exceed the capacity of mitochondrial respiration and induce an accumulation of toxic lipid metabolites, resulting in cardiac lipotoxicity and mitochondrial dysfunction [4] (Fig. 1). Adenosine monophosphateactivated protein kinase (AMPK) usually improves mitochondrial biogenesis through activation of peroxisome proliferator-activated receptor- $\gamma$ (PPAR- $\gamma$ ) coactivator- $1 \alpha$ (PGC-1 $\alpha$ ), which is a master metabolic regulator of mitochondrial biogenesis and respiratory function [4]. However, the PGC- $1 \alpha /$ AMPK signalling pathways involved in $\beta$ oxidation are impaired during the advanced stage of diabetic cardiomyopathy, thereby further contributing to mitochondrial dysfunction [4]. Moreover, increased fatty acid $\beta$-oxidation and mitochondrial dysfunction stimulates ROS generation and cardiac oxidative stress and inflammation. ROS in the human heart arise from mitochondrial generation as well as from NADPH oxidases, xanthine oxidase, uncoupling of NO synthase, microsomal P-450 enzymes and the arachidonic acid metabolism pathways [4]. The increased ROS induces further mitochondrial dysfunction and decreases fatty acid oxidation capacity, resulting in lipid accumulation, fibrosis, diastolic dysfunction and heart failure in individuals with diabetes.

Endoplasmic reticulum stress, impaired calcium handling and cardiomyocyte death The endoplasmic reticulum (ER) has an integral role in lipid synthesis, $\mathrm{Ca}^{2+}$ handling and protein folding and modification [28]. In diabetes, hyperglycaemia and insulin resistance induce ER stress, involving impaired $\mathrm{Ca}^{2+}$ handling and accumulation of unfolded proteins [29] (Fig. 1). $\mathrm{Ca}^{2+}$ enters cardiomyocytes through L-type $\mathrm{Ca}^{2+}$ channels and this induces cardiac $\mathrm{Ca}^{2+}$ release from sarcoplasmic reticulum through the ryanodine receptors [29]. Cardiac relaxation is normally maintained by sarcoplasmic reticulum reuptake of cardiomyocyte $\mathrm{Ca}^{2+}$, involving increased activity of the sarcoplasmic reticulum $\mathrm{Ca}^{2+}$ pump (SERCA), the $\mathrm{Na}^{+} / \mathrm{Ca}^{2+}$ exchanger and sarcolemmal $\mathrm{Ca}^{2+}$ ATPase [29]. However, in diabetic cardiomyopathy impaired $\mathrm{Ca}^{2+}$ reuptake results in increases in action potential duration and associated slowing of diastolic relaxation. The interaction of oxidative stress, ER stress and impaired $\mathrm{Ca}^{2+}$ handling promotes cardiomyocyte death via increased apoptosis, necrosis and autophagy [4] (Fig. 1). Elevated production of ROS increases the outer mitochondrial membrane permeability to further induce apoptosis [4], and excess uptake of $\mathrm{Ca}^{2+}$ increases $\mathrm{Ca}^{2+}$ overload to open mitochondrial permeability transition pores, also leading to cell apoptosis [30]. Impairment of normal autophagy, including defects in autophagosome and lysosome fusion in cardiomyocytes, is also involved in both diastolic and systolic cardiac dysfunction in diabetic cardiomyopathy [31].

Inappropriate activation of RAAS Activation of both the systemic and cardiac tissue RAAS despite a state of salt and volume excess in states of insulin resistance and hyperglycaemia plays an important role in the development of diabetic cardiomyopathy (Fig. 1). One study found that hyperglycaemia induced systemic RAAS activation, associated with increased vascular resistance and arterial pressure [32]. The importance of an activated RAAS in diabetes is supported by the observation that an antagonist of angiotensin II type 1 receptor produced a greater reduction in blood pressure in hyperglycaemic than in euglycaemic conditions [33]. Large randomised controlled trials have demonstrated that inhibition of aldosterone activity through blockade of the mineralocorticoid receptor decreases morbidity and mortality in both mild and moderately severe heart failure in individuals with diabetes [34]. Indeed, both angiotensin II and aldosterone directly prompt oxidative stress by increasing NADPH oxidase activity. Activation of the RAAS also induces systemic and cardiac insulin resistance through the mTOR-S6K1 signal transduction pathway [18]. Meanwhile, enhanced 
angiotensin II type 1 receptor and mineralocorticoid receptor signalling in the myocardium enhances the adaptive proinflammatory immune response and inflammation, including increases in leucocyte adhesion, cytokine expression and macrophage infiltration [5]. These abnormalities further induce activation of growth and profibrotic signalling pathways to result in cardiac fibrosis, diastolic dysfunction and heart failure.

Maladaptive immune modulation Activation of proinflammatory immune cells, including macrophages, dendritic cells and activated $\mathrm{T}$ lymphocytes, is also involved in diabetic cardiomyopathy [4]. Proinflammatory cytokines, such as TNF- $\alpha$, IL-6 and monocyte chemotactic protein 1 (MCP-1), contribute to cardiac oxidative stress and coronary artery dysfunction, ultimately leading to cardiac remodelling, fibrosis and diastolic dysfunction [4]. NF- $\mathrm{kB}$, a protein complex that controls transcription of DNA and inflammation cytokine production, is activated by fatty acids and hyperglycaemia in heart [4]. NF- $\mathrm{kB}$ not only induces the expression of proinflammatory cytokines, including TNF $\alpha$, IL-6, and IL-8, but also increases NLR family pyrin domain-containing 3 (NLRP3) inflammasome assembly and pro-caspase-1 autocleavage/activation, which in turn mediate pro-IL-1 $\beta$ processing and maturation [35]. Meanwhile, AGE/RAGE signalling also promotes NF-KB activation and therefore increases expression of proinflammatory cytokines and cardiac fibrosis [21]. Furthermore, macrophage proinflammatory M1 polarisation is increased whereas macrophage M2 anti-inflammatory response is inhibited in diabetic heart tissues [4]. While the proinflammatory macrophage M1 phenotype $\left(\mathrm{F} 4 / 80^{+} \mathrm{CD} 11 \mathrm{c}^{+}\right)$is generally associated with an increase in fibrosis, the macrophage $\mathrm{M} 2$ phenotype $\left(\mathrm{F} 4 / 80^{+} \mathrm{CD} 11 \mathrm{c}^{-}\right.$ $\mathrm{CD} 01^{+} \mathrm{Arg} 1^{+} \mathrm{CD} 206^{+}$) plays an important role in antiinflammatory responses and tissue repair [4]. Thus, systemic and local maladaptive immune function and inflammation responses play key roles in the cardiac remodelling and fibrosis that occur in diabetic cardiomyopathy (Fig. 1).

\section{Coronary endothelial dysfunction and dysregulation of}

exosomes The notion that dysfunctional coronary endothelial cells drive abnormal cardiac function has recently gained momentum [36, 37] (Figs 1, 2). Normally, NO, prostacyclin (prostaglandin $\mathrm{I}_{2}$ ) and endothelium-derived hyperpolarising factors (EDHFs) are released from coronary endothelial cells and exert beneficial effects including vasodilatation and reduced cardiac tissue inflammation [38]. In the early stages of insulin resistance and early diabetic cardiomyopathy, NOinduced vasodilatation is impaired whereas EDHF-mediated vasodilatation is generally preserved or even enhanced to maintain normal vascular tone [38]. However, in the later stages, both NO- and EDHF-induced vasodilatation may eventually become impaired, thereby promoting microvascular dysfunction and inflammation [38]. Further, reduction in bioavailable NO can lead to phosphorylation of the titin protein, thereby increasing the ratio of titin isoform N2B:N2BA expression and reducing cGMP-protein kinase G (PKG) signalling with resultant increased cardiac stiffness and impaired relaxation [4] (Fig. 2).

Exosomes are regarded as important mediators in intercellular communication and regulate normal physiological and pathophysiological effects [39]. These extracellular vesicles have a diameter of 30-90 nm and possess a variety of biological components including microRNAs (miRNAs), proteins and lipids [24]. Recent data suggest that dysregulation of exosomes is involved in diabetic cardiomyopathy (Fig. 1). For example, hypoxia, inflammation and hyperglycaemiainduced endothelial cell stress increase protein and messenger RNA content in endothelial cell-derived exosomes [40, 41]. Furthermore, the exosomes released from diabetic cardio myocytes can deliver detrimental components able to initiate endothelial cell dysfunction and impair angiogenesis [42]. Interestingly, heat shock protein 20-engineered exosomes exert beneficial effects via modulation of cardiomyocyte exosome secretion with restoration of normal cardiac function under hyperglycaemic conditions. These data suggest that exosomes and heat shock protein 20 might be novel therapeutic targets in the prevention of diabetic cardiomyopathy [42].

\section{Potential preventative and therapeutic strategies for diabetic cardiomyopathy}

It is clear that aerobic exercise and restriction of fat and refined carbohydrate intake are efficacious therapeutic methods for preventing and treating diabetic cardiomyopathy in individuals with the metabolic syndrome [4]. It is also clear that improving glycaemic control is a key factor in the prevention of diabetic cardiomyopathy and cardiovascular morbidity and mortality. For example, thiazolidinediones and metformin improve systemic and tissue insulin sensitivity, thus improving cardiomyocyte glucose uptake and cardiac function by activation of PPAR- $\gamma$ and AMPK, respectively [4].

Recently, incretin-based therapies have emerged as important agents in the treatment of type 2 diabetes and associated cardiovascular disease. For example, glucagon-like peptide 1 (GLP-1) receptor agonists and dipeptidyl peptidase-4 (DPP-4) inhibitors improve glycaemic disorders and reduce weight and are thus both regarded as among the best therapeutic options for type 2 diabetes [4]. One study further found that infusion of GLP-1 for 5 week improved left ventricular ejection fraction and functional status in diabetes patients who had chronic heart failure [43]. Generally, incretin-based therapies are regarded as being neutral on cardiovascular outcome profiles in individuals with type 2 diabetes. The major action underlying the effects of GLP-1 receptor antagonists and DPP-4 inhibitors is that GLP-1 increases the glucose sensitivity in 
pancreatic beta cells by upregulation of glucokinase and glucose transporter 2 [43]. However, a meta-analysis of randomised controlled trials suggested that glucose-lowering therapies, including peroxisome proliferator-activated receptor agonists and DPP-4 inhibitors, increase the risk of heart failure in individuals with (or who are at risk for) type 2 diabetes [44]. Thereby, the effect of intensive glycaemic control with various hypoglycaemic agents on cardiovascularassociated morbidity is not fully understood.

Inhibition of sodium-glucose cotransporter 2 reduced $\mathrm{HbA}_{1 \mathrm{c}}$ levels, blood pressure, weight, visceral adiposity and oxidative stress in individuals with type 2 diabetes [45]. In the EMPAREG OUTCOME trial involving individuals with type 2 diabetes, empagliflozin combined with exercise and other glucoselowering therapy was found to lower the rate of the primary outcome, a composite of death from cardiovascular causes, non-fatal myocardial infarction and non-fatal stroke [45].

Novel therapeutic drugs that reduce the AGE crosslinks formed in cardiovascular disease have been investigated recently. For example, alagebrium inhibited aortic stiffness and intra-aortic pulse pressure through breaking collagen crosslinks in hypertensive dogs [46]. However, alagebrium did not improve exercise tolerance in individuals with heart failure and systolic dysfunction, suggesting that it had a nonbeneficial effect in systolic heart failure [47]. Other therapies that play important roles in the prevention of diabetic cardiomyopathy, include metabolic modulators targeting mitochondrial oxidative stress, $\beta$-blockers and angiotensin II receptor antagonists [4].

\section{Conclusions}

Diabetic cardiomyopathy is manifested as abnormal cardiac structure and function in the absence of ischaemic or hypertensive heart disease in individuals with diabetes. Insulin resistance, hyperinsulinaemia and hyperglycaemia are independently associated with the development of cardiac dysfunction and heart failure. Pathophysiological abnormalities underlying diabetic cardiomyopathy include cardiac insulin resistance, glucotoxicity, mitochondrial dysfunction, oxidative stress, ER stress, impaired calcium handling, activation of systemic and tissue RAAS, impaired mitophagy and autophagy, coronary microvascular dysfunction and dysregulation of exosomes. However, there are still gaps in our knowledge of the different mechanisms involved in type 1/type 2 diabetesinduced cardiac dysfunction that remain to be investigated. There are no prospective clinical trials currently underway to confirm that type 1 diabetes or type 2 diabetes alone increases the risk of cardiac dysfunction and heart failure in the complete absence of other risk factors such as CAD, obesity and hypertension. Further studies are needed to understand the molecular mechanisms behind diabetic cardiomyopathy and to develop new preventative and therapeutic approaches.

Funding JRS received funding from NIH (R01 HL73101-01A and R01 HL107910-01) and the Veterans Affairs Merit System (0018), GJ received funding from the American Diabetes Association (Innovative Basic Science Award no. 1-17-IBS-201) and AW-C received funding from the Veterans Affairs Merit System (BX003391).

Duality of interest The authors declare that there is no duality of interest associated with this manuscript.

Contribution statement All authors were responsible for drafting and revising this review. All authors approved the version to be published.

\section{References}

1. Rubler S, Dlugash J, Yuceoglu YZ, Kumral T, Branwood AW, Grishman A (1972) New type of cardiomyopathy associated with diabetic glomerulosclerosis. Am J Cardiol 30:595-602

2. Kannel WB, Hjortland M, Castelli WP (1974) Role of diabetes in congestive heart failure: the Framingham study. Am J Cardiol 34: 29-34

3. Regan TJ, Lyons MM, Ahmed SS et al (1977) Evidence for cardiomyopathy in familial diabetes mellitus. J Clin Invest 60:884-899

4. Jia G, DeMarco VG, Sowers JR (2016) Insulin resistance and hyperinsulinaemia in diabetic cardiomyopathy. Nat Rev Endocrinol 12:144-153

5. Jia G, Habibi J, DeMarco VG et al (2015) Endothelial mineralocorticoid receptor deletion prevents diet-induced cardiac diastolic dysfunction in females. Hypertension 66:1159-1167

6. Raev DC (1994) Which left ventricular function is impaired earlier in the evolution of diabetic cardiomyopathy? An echocardiographic study of young type I diabetic patients. Diabetes Care 17:633-639

7. Lind M, Bounias I, Olsson M, Gudbjornsdottir S, Svensson AM, Rosengren A (2011) Glycaemic control and incidence of heart failure in 20,985 patients with type 1 diabetes: an observational study. Lancet 378:140-146

8. Brunvand L, Fugelseth D, Stensaeth KH, Dahl-Jorgensen K, Margeirsdottir HD (2016) Early reduced myocardial diastolic function in children and adolescents with type 1 diabetes mellitus a population-based study. BMC Cardiovasc Disord 16:103

9. Suran D, Sinkovic A, Naji F (2016) Tissue Doppler imaging is a sensitive echocardiographic technique to detect subclinical systolic and diastolic dysfunction of both ventricles in type 1 diabetes mellitus. BMC Cardiovasc Disord 16:72

10. Radovits T, Korkmaz S, Loganathan S et al (2009) Comparative investigation of the left ventricular pressure-volume relationship in rat models of type 1 and type 2 diabetes mellitus. Am J Physiol Heart Circ Physiol 297:H125-H133

11. Nichols GA, Gullion CM, Koro CE, Ephross SA, Brown JB (2004) The incidence of congestive heart failure in type 2 diabetes: an update. Diabetes Care 27:1879-1884

12. Iribarren C, Karter AJ, Go AS et al (2001) Glycemic control and heart failure among adult patients with diabetes. Circulation 103: 2668-2673

13. Stratton IM, Adler AI, Neil HA et al (2000) Association of glycaemia with macrovascular and microvascular complications of type 2 diabetes (UKPDS 35): prospective observational study. BMJ 321:405-412 
14. Adeghate E, Singh J (2014) Structural changes in the myocardium during diabetes-induced cardiomyopathy. Heart Fail Rev 19:15-23

15. Fang ZY, Prins JB, Marwick TH (2004) Diabetic cardiomyopathy: evidence, mechanisms, and therapeutic implications. Endocr Rev 25:543-567

16. Mytas DZ, Stougiannos PN, Zairis MN, Foussas SG, Pyrgakis VN, Kyriazis IA (2009) Diabetic myocardial disease: pathophysiology, early diagnosis and therapeutic options. J Diabetes Complicat 23: 273-282

17. Wang J, Song Y, Wang Q, Kralik PM, Epstein PN (2006) Causes and characteristics of diabetic cardiomyopathy. Rev Diabet Stud 3: 108-117

18. Kim JA, Jang HJ, Martinez-Lemus LA, Sowers JR (2012) Activation of mTOR/p70S6 kinase by ANG II inhibits insulinstimulated endothelial nitric oxide synthase and vasodilation. Am J Physiol Endocrinol Metab 302:E201-E208

19. van Heerebeek L, Hamdani N, Handoko ML et al (2008) Diastolic stiffness of the failing diabetic heart: importance of fibrosis, advanced glycation end products, and myocyte resting tension. Circulation 117:43-51

20. Lazo M, Halushka MK, Shen L et al (2015) Soluble receptor for advanced glycation end products and the risk for incident heart failure: the atherosclerosis risk in communities study. Am Heart J 170:961-967

21. Ma H, Li SY, Xu P et al (2009) Advanced glycation endproduct (AGE) accumulation and AGE receptor (RAGE) up-regulation contribute to the onset of diabetic cardiomyopathy. J Cell Mol Med 13:1751-1764

22. Makino A, Dai A, Han Y et al (2015) $O$-GlcNAcase overexpression reverses coronary endothelial cell dysfunction in type 1 diabetic mice. Am J Physiol Cell Physiol 309:C593-C599

23. Hu Y, Belke D, Suarez J et al (2005) Adenovirus-mediated overexpression of $O$-GlcNAcase improves contractile function in the diabetic heart. Circ Res 96:1006-1013

24. Lee TW, Bai KJ, Lee TI, Chao TF, Kao YH, Chen YJ (2017) PPARs modulate cardiac metabolism and mitochondrial function in diabetes. J Biomed Sci 24:5

25. Finck BN, Lehman JJ, Leone TC et al (2002) The cardiac phenotype induced by PPAR $\alpha$ overexpression mimics that caused by diabetes mellitus. J Clin Invest 109:121-130

26. Atkinson LL, Kozak R, Kelly SE, Onay Besikci A, Russell JC, Lopaschuk GD (2003) Potential mechanisms and consequences of cardiac triacylglycerol accumulation in insulin-resistant rats. Am J Physiol Endocrinol Metab 284:E923-E930

27. Mudaliar S, Alloju S, Henry RR (2016) Can a shift in fuel energetics explain the beneficial cardiorenal outcomes in the EMPA-REG OUTCOME study? A unifying hypothesis. Diabetes Care 39:11151122

28. Liu ZW, Zhu HT, Chen KL et al (2013) Protein kinase RNA-like endoplasmic reticulum kinase (PERK) signaling pathway plays a major role in reactive oxygen species (ROS)-mediated endoplasmic reticulum stress-induced apoptosis in diabetic cardiomyopathy. Cardiovasc Diabetol 12:158

29. Marciniak SJ, Ron D (2006) Endoplasmic reticulum stress signaling in disease. Physiol Rev 86:1133-1149

30. Yi CH, Vakifahmetoglu-Norberg H, Yuan J (2011) Integration of apoptosis and metabolism. Cold Spring Harb Symp Quant Biol 76: 375-387
31. Xie Z, Lau K, Eby B et al (2011) Improvement of cardiac functions by chronic metformin treatment is associated with enhanced cardiac autophagy in diabetic OVE26 mice. Diabetes 60:1770-1778

32. Miller JA, Floras JS, Zinman B, Skorecki KL, Logan AG (1996) Effect of hyperglycaemia on arterial pressure, plasma renin activity and renal function in early diabetes. Clin Sci (Lond) 90:189-195

33. Miller JA (1999) Impact of hyperglycemia on the renin angiotensin system in early human type 1 diabetes mellitus. J Am Soc Nephrol 10:1778-1785

34. Shen JZ, Young MJ (2012) Corticosteroids, heart failure, and hypertension: a role for immune cells? Endocrinology 153:5692-5700

35. Luo B, Li B, Wang W et al (2014) NLRP3 gene silencing ameliorates diabetic cardiomyopathy in a type 2 diabetes rat model. PLoS One 9:e104771

36. Paulus WJ, Tschope C (2013) A novel paradigm for heart failure with preserved ejection fraction: comorbidities drive myocardial dysfunction and remodeling through coronary microvascular endothelial inflammation. J Am Coll Cardiol 62:263-271

37. Franssen C, Chen S, Unger A et al (2016) Myocardial microvascular inflammatory endothelial activation in heart failure with preserved ejection fraction. JACC Heart Fail 4:312-324

38. Vincent MA, Clerk LH, Lindner JR et al (2004) Microvascular recruitment is an early insulin effect that regulates skeletal muscle glucose uptake in vivo. Diabetes 53:1418-1423

39. Westermeier F, Riquelme JA, Pavez M et al (2016) New molecular insights of insulin in diabetic cardiomyopathy. Front Physiol 7:125

40. de Jong OG, Verhaar MC, Chen Y et al (2012) Cellular stress conditions are reflected in the protein and RNA content of endothelial cell-derived exosomes. J Extracell Vesicles 1:18396

41. Garcia NA, Moncayo-Arlandi J, Sepulveda P, Diez-Juan A (2016) Cardiomyocyte exosomes regulate glycolytic flux in endothelium by direct transfer of GLUT transporters and glycolytic enzymes. Cardiovasc Res 109:397-408

42. Wang X, Gu H, Huang W et al (2016) Hsp20-mediated activation of exosome biogenesis in cardiomyocytes improves cardiac function and angiogenesis in diabetic mice. Diabetes 65:3111-3128

43. Sokos GG, Nikolaidis LA, Mankad S, Elahi D, Shannon RP (2006) Glucagon-like peptide-1 infusion improves left ventricular ejection fraction and functional status in patients with chronic heart failure. $J$ Card Fail 12:694-699

44. Udell JA, Cavender MA, Bhatt DL, Chatterjee S, Farkouh ME, Scirica BM (2015) Glucose-lowering drugs or strategies and cardiovascular outcomes in patients with or at risk for type 2 diabetes: a meta-analysis of randomised controlled trials. Lancet Diabetes Endocrinol 3:356-366

45. Zinman B, Wanner C, Lachin JM et al (2015) Empagliflozin, cardiovascular outcomes, and mortality in type 2 diabetes. N Engl J Med 373:2117-2128

46. Shapiro BP, Owan TE, Mohammed SF et al (2008) Advanced glycation end products accumulate in vascular smooth muscle and modify vascular but not ventricular properties in elderly hypertensive canines. Circulation 118:1002-1010

47. Hartog JW, Willemsen S, van Veldhuisen DJ et al (2011) Effects of alagebrium, an advanced glycation endproduct breaker, on exercise tolerance and cardiac function in patients with chronic heart failure. Eur J Heart Fail 13:899-908 